\title{
A Markovian Method for Predicting Trust Behavior in Human-Agent Interaction
}

\author{
David V. Pynadath \\ Ning Wang \\ Sreekar Kamireddy \\ pynadath@ict.usc.edu \\ nwang@ict.usc.edu \\ skamireddy@ict.usc.edu \\ University of Southern California Institute for Creative Technologies \\ Los Angeles, California, USA
}

\begin{abstract}
Trust calibration is critical to the success of human-agent interaction (HAI). However, individual differences are ubiquitous in people's trust relationships with autonomous systems. To assist its heterogeneous human teammates calibrate their trust in it, an agent must first dynamically model them as individuals, rather than communicating with them all in the same manner. It can then generate expectations of its teammates' behavior and optimize its own communication based on the current state of the trust relationship it has with them. In this work, we examine how an agent can generate accurate expectations given observations of only the teammate's trust-related behaviors (e.g., did the person follow or ignore its advice?). In addition to this limited input, we also seek a specific output: accurately predicting its human teammate's future trust behavior (e.g., will the person follow or ignore my next suggestion?). In this investigation, we construct a model capable of generating such expectations using data gathered in a humansubject study of behavior in a simulated human-robot interaction (HRI) scenario. We first analyze the ability of measures from a presurvey on trust-related traits to accurately predict subsequent trust behaviors. However, as the interaction progresses, this effect is dwarfed by the direct experience. We therefore analyze the ability of sequences of prior behavior by the teammate to accurately predict subsequent trust behaviors. Such behavioral sequences have shown to be indicative of the subjective beliefs of other teammates, and we show here that they have a predictive power as well.
\end{abstract}

\section{CCS CONCEPTS}

- Human-centered computing $\rightarrow$ Empirical studies in HCI; • Applied computing $\rightarrow$ Interactive learning environments.

\section{KEYWORDS}

trust,human-agent teams,probabilistic models

Permission to make digital or hard copies of all or part of this work for personal o classroom use is granted without fee provided that copies are not made or distributed for profit or commercial advantage and that copies bear this notice and the full citation on the first page. Copyrights for components of this work owned by others than the author(s) must be honored. Abstracting with credit is permitted. To copy otherwise, or republish, to post on servers or to redistribute to lists, requires prior specific permission and/or a fee.

Request permissions from Permissions@acm.org.

HAI '19, October 6-10, 2019, Kyoto, Japan

(C) 2019 Copyright is held by the owner/author(s). Publication rights licensed to ACM. ACM ISBN 978-1-4503-6922-0/19/10_..\$15.00

https://doi.org/10.1145/3349537.3351905
ACM Reference Format:

David V. Pynadath, Ning Wang, and Sreekar Kamireddy. 2019. A Markovian Method for Predicting Trust Behavior in Human-Agent Interaction. In Proceedings of the 7th International Conference on Human-Agent Interaction (HAI '19), October 6-10, 2019, Kyoto, Japan. ACM, New York, NY, USA, 7 pages. https://doi.org/10.1145/3349537.3351905

\section{INTRODUCTION}

Trust calibration is critical to the success of human-agent interaction (HAI) [7, 9]. To maximize the performance of human-agent teams, people should trust an agent teammate to perform a task autonomously when it is capable of doing so and distrust it otherwise. Failure to do so results in disuse and misuse, respectively, of the agent $[7,14]$. Research has shown that people will more accurately trust an agent if they have a more accurate understanding of its decision-making process [5].

However, individual differences are ubiquitous in people's trust relationships with autonomous systems (e.g., [5, 6, 19]), and heterogeneous human teammates will not all be able to achieve trust calibration with an agent that communicates the same with all of them. To assist in its teammates' trust-calibration process, an agent must first dynamically model them as individuals and then generate expectations of their behavior and explain its decision making based on the current state of the trust relationship it has with them. To tackle this subproblem of trust calibration, we can leverage the wide range of methods for recognizing hidden states of other agents [20], even trust-relevant hidden states [1]. Our focus here is more similar to affect recognition [25], rather than recognition of domain-level plans and intentions. Furthermore, our specific recognition problem limits the agent's online observations to only trust-related behavior (e.g., did the person follow or ignore its advice?). In addition to this difference in input, we also seek a specific output: accurately predicting its human teammate's future trust behavior (e.g., will the person follow or ignore my next suggestion if I do not explain it?). Successfully performing such trust recognition will enable the agent to generate expectations as to its teammates' behavior in response to its own, thus supporting an informed choice of its own behavior.

In this investigation, we construct a model capable of generating such expectations using data gathered in a human-subject study of behavior in a simulated human-robot interaction (HRI) scenario. We first analyze the ability of measures from a pre-survey on trust-related traits to accurately predict subsequent trust behaviors. However, as the interaction progresses, this effect is dwarfed by the 
direct experience. We therefore analyze the ability of sequences of prior behavior by the teammate to accurately predict subsequent trust behaviors. Such behavioral sequences have shown to be indicative of the subjective beliefs of other teammates [15], and we show here that they have a predictive power as well.

\section{HUMAN-AGENT INTERACTION SCENARIO}

\subsection{Experimental Testbed}

We gathered our data in the context of an online HAI testbed [22] in which a human teammate works with a different robot across eight reconnaissance missions (see Figure 1). Each mission requires the human teammate to search 15 buildings in a different town. The virtual robot serves as a scout: it scans the buildings for potential danger and relays its findings. We give the robot noisy observations, so that its assessment of the safety/danger of a building is correct in only 12 out of the 15 buildings in each mission. We limited the mistakes made to be only false negatives for a previous analytical goal, and these mistakes are sufficient for disincentivizing misuse of the robot through overreliance $[7,14]$. The methodology of this investigation would also work for false positives, and we plan to gather additional data with that case in the future.

The human must choose between entering a building with or without protective gear. If there is danger inside the building, the human will be "fatally injured" (incurring a 3-minute time penalty) if not wearing the protective gear. If the human teammate fails to enter all 15 buildings within 10 minutes, the mission is a failure. Four buildings in each mission contain threats (a different four in each mission sequence), so entering all of them without protective gear almost guarantees mission failure. On the other hand, it takes time to put on and take off protective gear (20 seconds each). Therefore, putting on the protective gear for all 15 buildings also leads to mission failure. We thus also disincentivize disuse of the robot $[7,14]$, so that people consider the robot's findings to make a more informed decision as to wearing or not wearing the protective gear.

The virtual robot chooses a recommendation as to whether its teammate should or should not put on protective gear by following a policy generated from a Partially Observable Markov Decision Process (POMDP) [3] (details of the POMDP are presented in [23]). The participant needs to decide only whether to follow or ignore the robot's findings (safe/dangerous), before pressing a button to enter/exit the room. In the testbed implementation for the current study, the participant works with a different robot for each mission. Each of the eight robot represents a different combination along the following three binary dimensions:

Confidence: Half of the robots provide an assessment of a building's safety as being safe or dangerous, with no additional information (e.g., "I have finished surveying the doctor's office. I think the place is safe."). The other half of the robots augment their decisions with additional information that should help its teammate better understand its ability (e.g., decision-making), one of the key dimensions of trust [10]. These robots give a confidence-level elaboration that augments the decision message with additional information about the robot's uncertainty in its decision. One example of a confidence-level elaboration would be: "I have finished surveying the Cafe. I think the place is dangerous. I am $86 \%$ confident about this assessment." The robot uses its current probabilistic belief state (derived from its POMDP model of the world) to fill in the percentage confidence. In the buildings in which the robot's assessment is incorrect (3 out of 15 each mission), the confidence level that it computes and communicates is lower than it is for the other buildings.

Acknowledgment: Half of the robots send an additional message every time they make an assessment that turns out to be incorrect; the other half do not send any such message. In each mission, the team searches 15 buildings, and the robot makes an incorrect assessment of three of them. An example of the robot's acknowledgement would be "It seems that my assessment of the informant's house was incorrect. I will update my algorithms when we return to base after the mission." This acknowledgment is inspired by a prior investigation in organizational trust that found that an acknowledgement of a mistake, paired with a promise to improve, would improve trust under certain conditions [18]. One can view this action as an attempt by the robot at trust repair, which plays a critical role in maintaining long-term organizational trust [8].

Embodiment: Half of the robots look like a robotic dog, with ears, nose and highlighted eyes, suggesting possibly embedded sound, NBC, and vision sensors. The other half look like a stereotypical "robot-looking" robot (depicted in Figure 1). This variation is motivated by studies showing that doglike robots are treated differently than those with a more traditionally robotic appearance $[4,13]$.

For the purposes of this investigation, the critical difference is whether or not the robot provides the confidence-level elaboration on its assessment. The acknowledgment and embodiment had negligible impact in general.

\subsection{Experimental Design}

53 participants from a higher-education military school in the United States were able to complete the background survey and all eight missions. Participants were awarded extra course credit for their participation. The background survey items of particular interest to this investigation concern the individual's predisposition to trust [11], propensity to trust [12], complacency potential [16], negative attitude towards robots (NARS) [21], and uncertainty response [2]. These items provide information as to people's prior biases, which could in turn influence their initial trust behavior. Based on observations made in prior work $[15,24]$, this influence can account for the more diverse behaviors that people exhibit during their first interaction with an agent (the first mission in our experiment).

We also expect there to be minimal influence of such personal characteristics in later missions, when direct experience with the robot is the primary determinant of the trust relationship. We capture this direct experience in the logs of the participants' behavior in the system, allowing us to extract the decision sequence of each participant aligned with the agent's own behavior. We seek to quantify the degree to which these observable behaviors can be used by an agent to generate accurate expectations of the teammate's future behavior. 


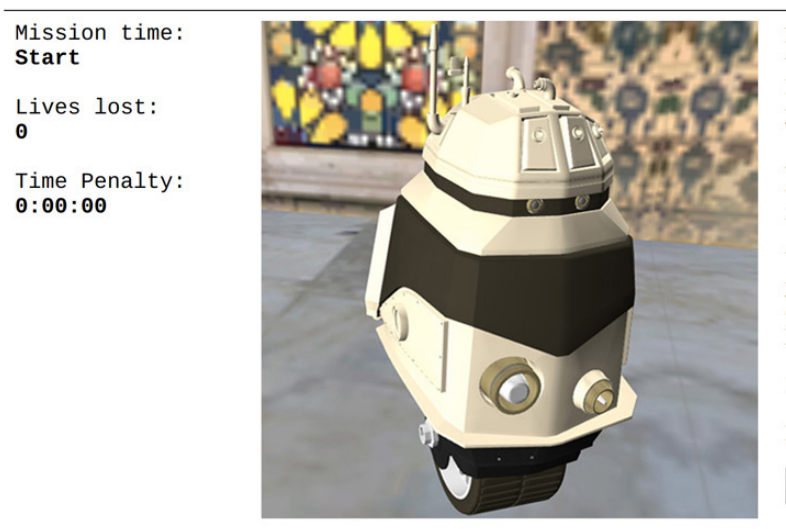

Robot: Welcome to Market City. I am your robot teammate for this mission. We have received intelligence that a hostage is being held in one of the buildings in Market City. Our mission is to gather intelligence on Market City, including the whereabouts of the hostage. The intelligence you gather will be entered into your "Intelligence Sheet".

As your teammate, I will survey each building for potential threats in advance, and send you messages about my findings. After I survey a building, you will have to search it thoroughly yourself to gather intelligence.

You may encounter threats during your mission. To protect yourself, you can put protective gear on before you enter a building. It takes 20 seconds to put protective gear on and another 20 seconds to take it back off. But if you're not wearing protective gear when you encounter a threat, you will lose a life. For each life lost, 3 minutes will be added to the mission completion time at the end of the mission.

If the mission completion time is longer than 10 minutes, it will be considered mission failure.

Let's get started! First things first, I will check out the Warehouse.

Start mission

Figure 1: Screenshot of HRI testbed with HTML front-end.

\subsection{Data Structure}

We can characterize each mission as 15 episodes of the HAI. In each episode, there is a "ground truth", $g$, representing whether the building is actually safe (S) or unsafe (U). Then, there is the robot's recommendation, $r$, which is an assessment of the building's safety, and which can be correct (C) or incorrect (I). Finally, there is the human teammate's behavior, $h$, to either follow (F) or ignore (I) the robot's assessment. The mission then proceeds to the next building. Throughout a mission, the robot's decision elaboration, $e$, is either a confidence-level message $(\mathrm{C})$ or no elaboration $(\mathrm{N})$.

We can therefore characterize the history of the overall HAI in terms of subsequences of these variables. We then try to predict the human teammate's subsequent trust behavior from this history. If we limit our history to just the building at the current time $t$ (which we call a window of length 0 ), there are four possible states for $g_{t}$ and $r_{t}$ (SC, SI, UC, UI) from which we can try to predict the teammate's trust behavior, $h_{t}$. If we extend the window to be length 1 , there are eight possible states for the previous episode at time $t-1\left(g_{t-1} r_{t-1} h_{t-1} \in\{\mathrm{SCF}, \mathrm{SCI}, \mathrm{SIF}, \mathrm{SII}, \mathrm{UCF}, \mathrm{UCI}, \mathrm{UIF}, \mathrm{UII})\right.$, leading to 32 possible states for the overall prediction of $h_{t}$. Extending the window again to include the $t-2$ episode, we have 256 possible states for predicting $h_{t}$, and so on. If the agent used the entire history to predict the person's behavior in the fifteenth (and final) building, there would be $>10^{13}$ possible states. Our available data are nowhere near sufficient for providing meaningful values in a table of that size, so we keep our windows much shorter in this investigation.

\section{PREDICTING INITIAL TRUST BEHAVIOR}

During the first mission with a new teammate, our agent does not have direct experience to inform its expectations. However, it does have the background survey responses to inform it as to its teammate's personality characteristics. We therefore first examine whether any of the measures available through the background survey are indicative of trends in the person's trust behavior in
Mission 1. To do so, we aggregate the participants' Mission 1 behaviors into histories of length 0,1 , and 2 , and then measure the frequency of following or ignoring the robot's assessment. For example, for length 0 , we have $53 \cdot 15=795$ total episodes that we can characterize by sequences like UC(C) and SI(N) (where the symbol in parentheses indicates whether confidence-level information was provided or not).

We break down the people in each sequence according to the background survey metrics:

- Complacency potential

- Cognitive uncertainty

- Desire for change

- Emotional uncertainty

- NARS

- Predisposition to trust

- Propensity to trust

For each metric and sequence combination, we divide the people into two groups based on whether they score above or below the median value for that metric (across the whole pool). We then measure the frequency of follow vs. ignore behaviors by each group. We can thus observe, for example, that in the UC(N) sequence (an unsafe building that the robot correctly assesses, but does not elaborate on), the people with a high Predisposition to trust exhibit the same percentage of follow behaviors as those with low values.

Table 1 shows the ten sequence-measure combinations that have the highest-magnitude impacts on the frequency of following the robot's assessment, when aggregated over windows of length 0 . The difference is the frequency of following in the given sequence for those who scored high on the measure minus the same frequency for those who scored low. Thus, a positive difference indicates that participants who scored high on the measure were more likely to follow, while a negative difference indicates the opposite.

One observation we can make (which is also true if we examine the results of windows of lengths 1 and 2) is that the type of building that shows the biggest effect of the trust measures is $\mathrm{UC}(\mathrm{N})$, i.e., one that is unsafe and that the robot correctly identifies as unsafe, 


\begin{tabular}{|l|c|r|}
\hline Measure & Sequence & Difference \\
\hline \hline Propensity to trust & $\mathrm{UC}(\mathrm{N})$ & 0.50 \\
\hline Complacency potential & $\mathrm{UC}(\mathrm{C})$ & -0.44 \\
\hline Emotional uncertainty & $\mathrm{UC}(\mathrm{N})$ & -0.35 \\
\hline Desire for change & $\mathrm{UC}(\mathrm{N})$ & -0.35 \\
\hline Cognitive uncertainty & $\mathrm{UC}(\mathrm{N})$ & -0.35 \\
\hline NARS & $\mathrm{UC}(\mathrm{N})$ & -0.19 \\
\hline Emotional uncertainty & $\mathrm{UI}(\mathrm{N})$ & 0.17 \\
\hline Desire for change & $\mathrm{UI}(\mathrm{N})$ & 0.17 \\
\hline Cognitive uncertainty & $\mathrm{UI}(\mathrm{N})$ & 0.17 \\
\hline NARS & $\mathrm{UI}(\mathrm{N})$ & -0.15 \\
\hline
\end{tabular}

Table 1: Sequence-Measure combinations with the largestmagnitude impact on trust behaviors for windows of length o

\begin{tabular}{|l|c|r|}
\hline Measure & Sequence & Difference \\
\hline \hline Propensity to trust & SCF UC(N) & 0.71 \\
\hline Emotional uncertainty & SCF UC(N) & -0.54 \\
\hline Cognitive uncertainty & SCF UC(N) & -0.54 \\
\hline Desire for change & SCF UC(N) & -0.54 \\
\hline Predisposition to trust & SCI SC(N) & 0.48 \\
\hline Propensity to trust & SCI UI(N) & -0.44 \\
\hline Complacency potential & SCF UC(C) & -0.42 \\
\hline Emotional uncertainty & SCI SC(N) & 0.39 \\
\hline Propensity to trust & SCI SC(N) & -0.39 \\
\hline Cognitive uncertainty & SCI SC(N) & 0.39 \\
\hline
\end{tabular}

Table 2: Sequence-Measure combinations with the largestmagnitude impact on trust behaviors for windows of length 1

but provides no confidence measure. Unsafe buildings are the minority in our scenario, and those that the robot correctly identifies are even rarer. Without any first-hand knowledge of the robot's reliability in these situations, people seem to fall back to their initial biases to interpret such seemingly anomalous events. Perhaps not surprisingly, the bias that has the strongest effect in this table is Propensity to trust, in that those who score higher on this measure are more likely to follow the robot. There are weaker effects for other biases in this situation as well: Emotional uncertainty (low means more likely), Cognitive uncertainty (low means more likely), and Desire for change (low means more likely).

Table 2 shows a similar table, but aggregated over windows of length 1 . Here, the sequences include the previous episode, including whether the teammate followed or ignored the agent's previous assessment. We again see that the top impacts occur when the current room (the second set of symbols in the sequence) is correctly identified as unsafe by the agent. Furthermore, within such rooms, the strongest effect is in people who followed previously. In contrast, people who ignored the agent in the previous episode (sequence SCI UC(N)) showed differences that were roughly a third as big as the ones that appear at the top of Table 2 .

\begin{tabular}{|l|r|}
\hline Sequence & $P(h=\mathrm{F})$ \\
\hline \hline $\mathrm{SC}(\mathrm{C})$ & 0.97 \\
\hline $\mathrm{SC}(\mathrm{N})$ & 0.90 \\
\hline $\mathrm{UC}(\mathrm{C})$ & 0.62 \\
\hline $\mathrm{UC}(\mathrm{N})$ & 0.71 \\
\hline $\mathrm{UI}(\mathrm{C})$ & 0.18 \\
\hline $\mathrm{UI}(\mathrm{N})$ & 0.92 \\
\hline
\end{tabular}

Table 3: Frequency of following under sequences of length 0

We can also examine the situations under which the measures do not engender any difference in the subsequent trust behavior. Many of these situations are very rare, but the sequence SCF UC(C) did occur 26 times, without any observable impact of Cognitive Uncertainty, Desire for change, or Emotional uncertainty on trust behavior. In this situation, the person followed a correct assessment of safety and is now observing the robot assess the current building as unsafe with an associated confidence level. These three biases show no influence on the subsequent trust behavior. In contrast, we can see in Table 2 that people with a low complacency potential are more likely to put on protective gear in this same situation.

Overall, Propensity to trust has the largest impact under all of our window lengths, well above Desire for change and the two uncertainty response measures. For windows length 1, the impact of Propensity to trust was nearly twice as much as that of NARS, which had the smallest impact. That Propensity to trust has the largest impact is perhaps not surprising, but it is surprising that Predisposition to trust had the smallest impact for widows of length 0 and the second-smallest for length 1 . Further investigation into the individual survey items is needed to better understand this difference. Such investigation might also lead to identifying an aggregate measure that has even more impact, although these existing aggregate measures are clearly informative as to predicting people's initial trust behaviors.

\section{PREDICTING SUBSEQUENT TRUST BEHAVIOR}

As observed in prior work [15], this highly variable trust behavior is only transient, and people start conforming to a smaller set of possible behaviors as they interact with the agent and get a better understanding of how it works. In this more stable interaction, the agent can use just the sequence information to predict its teammate's behaviors. At this point, we would expect the various background measures to become just that-background.

Table 3 shows the frequency table for length 0 windows. We immediately see that people typically follow the robot's recommendation. The only situation under which people regularly ignore it is $\mathrm{UI}(\mathrm{C})$, i.e., when the robot incorrectly assesses an unsafe room as safe, but provides a confidence level that is sufficient for most participants to realize that it is in error. We can also see that when the robot correctly identifies an unsafe building, people generally follow its recommendation, but not as reliably as they do when it correctly identifies a safe building. 


\begin{tabular}{|l|r|}
\hline Sequence & $P(h=\mathrm{F})$ \\
\hline \hline UCF SC(C) & 1 \\
\hline UCI SC(C) & 1 \\
\hline UIF SC(C) & 0.99 \\
\hline UII SC(C) & 0.99 \\
\hline UCI SC(N) & 0.98 \\
\hline UIF UI(N) & 0.98 \\
\hline SCF SC(C) & 0.97 \\
\hline UCF SC(N) & 0.97 \\
\hline SCF SC(N) & 0.92 \\
\hline SCF UI(N) & 0.92 \\
\hline UIF SC(N) & 0.89 \\
\hline SCI SC(C) & 0.80 \\
\hline UIF UI(C) & 0.74 \\
\hline UII UI(N) & 0.71 \\
\hline SCI UI(N) & 0.69 \\
\hline SCF UC(N) & 0.68 \\
\hline SCI UC(N) & 0.68 \\
\hline SCI SC(N) & 0.66 \\
\hline SCF UC(C) & 0.62 \\
\hline UII SC(N) & 0.59 \\
\hline SCI UC(C) & 0.33 \\
\hline SCI UI(C) & 0.27 \\
\hline SCF UI(C) & 0.16 \\
\hline UII UI(C) & 0.12 \\
\hline
\end{tabular}

Table 4: Frequency of following under sequences of length 1

Table 4 shows a similar table for windows of length 1 . We see that low-following situation UI(C) from Table 3 exhibits consistently low following frequency even when considering the possible prior episode. In particular, if the agent correctly identified the previous building as safe, then the frequency of following the robot's incorrect assessment of the current unsafe building is $16 \%$ or $27 \%$, depending on whether the teammate followed or ignored, respectively, the robot's previous assessment. However, if the robot incorrectly identified the previous building as safe, then people who followed that incorrect assessment were $74 \%$ likely to follow the robot's current one as well. People who ignored the robot's previous incorrect assessment were more likely to ignore the robot's current one than the other three groups. The negative interpretation of such behavior is that the participants have grown to generally distrust the robot. The positive interpretation is that the participants have correctly calibrated their trust in the robot, as they are able to correctly ignore its incorrect assessments.

Although these tables are aggregated from the participants' actual behaviors, it is not clear how well they represent that behavior. To quantify the accuracy of this summarization, we can use these tables to go back and predict what the participants would have done. In other words, we compare each participant's actual trust behavior in each episode against what our frequency tables (of different lengths) would predict given the same history. We simplify the prediction here to yield follow if the frequency is $\geq 50 \%$,

\begin{tabular}{|r||r|r|r|r|}
\hline Correct Missions & Length 0 & Length 1 & Length 2 & Length 3 \\
\hline \hline 0 & 5 & 4 & 2 & 0 \\
\hline 1 & 8 & 8 & 8 & 6 \\
\hline 2 & 7 & 5 & 6 & 7 \\
\hline 3 & 11 & 13 & 12 & 13 \\
\hline 4 & 8 & 9 & 11 & 9 \\
\hline 5 & 7 & 7 & 5 & 7 \\
\hline 6 & 3 & 3 & 5 & 6 \\
\hline 7 & 2 & 2 & 2 & 3 \\
\hline 8 & 1 & 1 & 1 & 1 \\
\hline
\end{tabular}

Table 5: Histogram of missions correctly predicted per person by tables of varying history lengths

\begin{tabular}{|r||r|r|r|r|}
\hline \% Episodes & Length 0 & Length 1 & Length 2 & Length 3 \\
\hline \hline $70-74$ & 1 & 0 & 0 & 0 \\
\hline $75-79$ & 3 & 4 & 3 & 2 \\
\hline $80-84$ & 5 & 5 & 3 & 5 \\
\hline $85-89$ & 10 & 10 & 18 & 11 \\
\hline $90-94$ & 17 & 20 & 15 & 22 \\
\hline $95-99$ & 15 & 12 & 12 & 11 \\
\hline 100 & 1 & 1 & 1 & 1 \\
\hline \hline Overall \% & 90.3 & 90.5 & 90.6 & 91.3 \\
\hline
\end{tabular}

Table 6: Histogram of percentage of episodes correctly predicted per person by tables of varying history lengths

although in general people would use a threshold that reflected their expected utility of following the robot's recommendation.

In doing so, we observe that there is one participant whose trust behaviors are predicted perfectly, across all eight missions, by the tables for windows of lengths $0,1,2$, and 3 (we did not evaluate tables with higher lengths). On the other hand, this means that the agent would have made at least one prediction error for all of the other participants. For a more fine-grained look, Table 5 summarizes an analysis of correctness over individual missions. From this table, we see that predictions using histories of length 3 have an entry of 0 in the first row (0 Correct Missions), meaning that for every participant, this model predicted at least one mission of their trust behaviors completely right. In contrast, shortening the history length leads to more participants never having the agent be able to correctly predict their trust behaviors all the way through.

In general, Table 5 shows a slight improvement as the window length increases. Of course, if we can be forgiving of inaccurate predictions, then we can examine the percentage of accurate predictions across participants, as presented in Table 6. The worst performance is by the 0-length frequency table, which achieves $72.5 \%$ accuracy on one particular user; none of the other tables achieve less than $75 \%$ on any participant. We see that, even though all of these participants' behaviors are included in these tables, many of them will have a sizable percentage of their trust behaviors incorrectly predicted by the agent.

The bottom row of Table 6 gives the overall accuracy across all participants and episodes, which increases very slowly with 
the window length, but is consistently around $90 \%$. Given that we are evaluating the accuracy on the same data used to generate the tables, the fact that a $\sim 10 \%$ error remains suggests that the model is very coarse, even as a summary of the existing data. As a baseline, we trained a recurrent neural network $(\mathrm{RNN})$ on a portion of the data and tested it on the rest, producing a measure of $84.3 \%$ accuracy [17]. The RNN provides a machine-learning method appropriate for classification based on input sequences (such as in our data), although it does not have the advantage of the domain knowledge that informs our model's structural assumptions.

While our frequency tables are not as powerful a representation as an RNN, they do provide a transparent representation that allows us to structure the participants' behavior in a way to reveal insights. In particular, the overall accuracy number is less important in this domain as is understanding which participants' trust behaviors are more easily predicted from their history than others. Having identified these participants, we can go back to our background survey measures to see whether they give us leverage in trying to distinguish these types of participants. Our current data are too sparse to provide strong evidence for such connections, so gathering further data is a must here.

\section{CONCLUSION}

We have thus been able to identify a relatively simple probabilistic model that shows promise in being able to summarize and predict human trust behaviors based on background personality traits and limited history information from an HAI scenario. We are in the process of testing the models generated here on data gathered in a separate study. This further analysis will provide a measure of the generalizability of the findings using this method.

Some of the trends observed here may not generalize, as they may be due to a trend in the overall scenario: most of the buildings are safe, and the agent never makes false positives. We are in the process of enriching the scenario to more consistently cover the possible situations. The resulting data should provide more thorough frequencies to inform the prediction model.

The model itself also needs enrichment. The current model provides a great deal of transparency, in that it is clear to understand what is meant and what the implications are for agent design. While an RNN is most likely overkill for this domain, other probabilistic models (e.g., hidden Markov models) could do a better job of improving with data.

Of course, the prediction is only a means to the agent's ultimate end of a decision as to how to communicate its assessment to its teammate. It must thus apply the probability of following vs. ignoring within an evaluation of possible communication strategies. The current tables quantify the impact of confidence-level elaboration vs. nothing, but more alternative elaborations and explanations are necessary. Fortunately, we have implemented alternate explanations within our testbed, and newly gathered data can be immediately fed through the process outlined here to generate prediction probabilities over these alternatives. The agent can then use these probabilities (as well as utilities for following vs. ignoring) to determine the optimal explanation to give. The result of this decision-making will be an agent that dynamically alters its communication strategy to maximize the performance of the human-agent team.

\section{ACKNOWLEDGMENTS}

This work was sponsored by the U.S. Army Research, Development, and Engineering Command (RDECOM) and the U.S. Army Research Laboratory.

\section{REFERENCES}

[1] Robin Cohen and Rino Falcone Timothy Norman (Eds.). 2016. International Workshop on Trust in Agent Societies.

[2] Veronica Greco and Derek Roger. 2001. Coping with uncertainty: The construction and validation of a new measure. Personality and individual differences 31,4 (2001), 519-534.

[3] Leslie Pack Kaelbling, Michael L. Littman, and Anthony R. Cassandra. 1998. Planning and acting in partially observable stochastic domains. Artificial intelligence 101, 1 (1998), 99-134.

[4] A. Kerepesi, E. Kubinyi, G.K. Jonsson, M.S. Magnusson, and A. Miklosi. 2006. Behavioural comparison of human-animal (dog) and human-robot (AIBO) interactions. Behavioural processes 73, 1 (2006), 92-99.

[5] John Lee and Neville Moray. 1992. Trust, control strategies and allocation of function in human-machine systems. Ergonomics 35, 10 (1992), 1243-1270.

[6] John D Lee and Neville Moray. 1994. Trust, self-confidence, and operators' adaptation to automation. International journal of human-computer studies 40, 1 (1994), 153-184.

[7] John D. Lee and Katrina A. See. 2004. Trust in automation: Designing for appropriate reliance. Human factors 46, 1 (2004), 50-80.

[8] R. J. Lewicki. 2006. Trust, trust development, and trust repair. In The handbook of conflict resolution: Theory and practice, $\mathrm{M}$. Deutsch, P. T. Coleman, and E. C. Marcus (Eds.). Wiley Publishing, San Francisco, CA, 92-119.

[9] Michael Lewis, Katia Sycara, and Phillip Walker. 2017. The role of trust in humanrobot interaction. In Foundations of Trusted Autonomy, Hussein A. Abbass, Jason Scholz, and Darryn J Reid (Eds.). Springer-Verlag, Basel, Switzerland, 135-159.

[10] Roger C. Mayer, James H. Davis, and F. David Schoorman. 1995. An integrative model of organizational trust. Academy of Management Review 20, 3 (1995), 709-734.

[11] D Harrison McKnight, Vivek Choudhury, and Charles Kacmar. 2002. Developing and validating trust measures for e-commerce: An integrative typology. Information Systems Research 13, 3 (2002), 334-359.

[12] S.L. McShane. 2014. Propensity to trust scale. (2014).

[13] Gail F. Melson, Peter H. Kahn, Alan Beck, Batya Friedman, Trace Roberts, Erik Garrett, and Brian T. Gill. 2009. Children's behavior toward and understanding of robotic and living dogs. Fournal of Applied Developmental Psychology 30, 2 (2009), 92-102.

[14] Raja Parasuraman and Victor Riley. 1997. Humans and automation: Use, misuse, disuse, abuse. Human factors 39, 2 (1997), 230-253.

[15] David V. Pynadath, Ning Wang, Ericka Rovira, and Michael J. Barnes. 2018. Clustering Behavior to Recognize Subjective Beliefs in Human-Agent Teams. In Proceedings of the International foint Conference on Autonomus Agents and MultiAgent Systems. ACM, New York, NY, 1495-1503. http://ifaamas.org/Proceedings/ aamas2018/pdfs/p1495.pdf

[16] Jennifer Marie Ross. 2008. Moderators of trust and reliance across multiple decision aids. Ph.D. Dissertation. University of Central Florida.

[17] David E Rumelhart, Geoffrey E Hinton, Ronald J Williams, et al. 1988. Learning representations by back-propagating errors. Cognitive Modeling 5, 3 (1988), 1.

[18] Maurice E. Schweitzer, John C. Hershey, and Eric T. Bradlow. 2006. Promises and lies: Restoring violated trust. Organizational behavior and human decision processes 101, 1 (2006), 1-19.

[19] Indramani L Singh, Robert Molloy, and Raja Parasuraman. 1993. Individual differences in monitoring failures of automation. The fournal of General Psychology 120, 3 (1993), 357-373.

[20] Gita Sukthankar, Robert P. Goldman, Christopher Geib, David V. Pynadath, and Hung Hai Bui (Eds.). 2014. Plan, Activity, and Intent Recognition: Theory and Practice. Elsevier, Waltham, MA.

[21] Dag Sverre Syrdal, Kerstin Dautenhahn, Kheng Lee Koay, and Michael L Walters. 2009. The negative attitudes towards robots scale and reactions to robot behaviour in a live human-robot interaction study. In Adaptive and Emergent Behaviour and Complex Systems: Proceedings of the 23rd Convention of the Society for the Study of Artificial Intelligence and Simulation of Behaviour. SSAISB.

[22] Ning Wang, David V. Pynadath, and Susan G. Hill. 2015. Building trust in a human-robot team. In Interservice/Industry Training, Simulation and Education Conference.

[23] Ning Wang, David V. Pynadath, and Susan G. Hill. 2016. The impact of POMDPgenerated explanations on trust and performance in human-robot teams. In 
International Conference on Autonomous Agents and Multiagent Systems. ACM, New York, NY, 997-1005.

[24] Ning Wang, David V. Pynadath, Susan G. Hill, and Chirag Merchant. 2017. The dynamics of human-agent trust with POMDP-generated explanations. In International Conference on Intelligent Virtual Agents. Springer, 459-462.
[25] Zhihong Zeng, Maja Pantic, Glenn I. Roisman, and Thomas S. Huang. 2009. A survey of affect recognition methods: Audio, visual, and spontaneous expressions. IEEE Transactions on Pattern Analysis and Machine Intelligence 31, 1 (2009), 39-58. 